Research Article

\title{
Logistic Regression Analysis of Relationship between Changes of Cerebrospinal Fluid and Communicating Hydrocephalus after Decompressive Craniectomy in Craniocerebral Injury under Computed Tomography Images
}

\author{
Long Sun $\mathbb{D}$, Haitao Wang $\mathbb{D}$, and Jian Huang $\mathbb{1}$ \\ Department of Neurosurgery, Linyi Central Hospital, 17 Jiankang Road, Yishui County, Linyi 276400, Shandong, China \\ Correspondence should be addressed to Jian Huang; lysyszxyy@163.com
}

Received 7 April 2021; Revised 3 June 2021; Accepted 18 June 2021; Published 1 July 2021

Academic Editor: Gustavo Ramirez

Copyright $\odot 2021$ Long Sun et al. This is an open access article distributed under the Creative Commons Attribution License, which permits unrestricted use, distribution, and reproduction in any medium, provided the original work is properly cited.

\begin{abstract}
This paper aimed to explore the application value of CT imaging in the correlation analysis of communicating hydrocephalus $(\mathrm{CH})$ after decompressive craniectomy (DC) of craniocerebral injury (CI). 410 patients with craniocerebral trauma who were admitted to the hospital from October 2015 to October 2019 were taken as subjects, among which 130 patients suffered from $\mathrm{CH}$. All patients underwent craniocerebral CT examination, and logistic regression was applied to analyze the risk factors of hydrocephalus in CI and hydrocephalus after DC surgery. The results showed that the coma time $(\mathrm{OR}=5.1283, P=0.001)$, subarachnoid hemorrhage $(\mathrm{SAH})(\mathrm{OR}=7.6543, P=0.020)$, Glasgow Coma Scale (GCS) score no more than 8 points $(\mathrm{OR}=3.5480, P=0.001)$, intraventricular hemorrhage $(\mathrm{OR}=2.2653, P=0.003)$, cerebral contusion and laceration $(\mathrm{OR}=1.036$, $P=0.002)$, and subdural hemorrhage $(\mathrm{OR}=2.4376, P=0.001)$ were independent risk factors for $\mathrm{CH}$. Bilateral $\mathrm{DC}(\mathrm{OR}=15.342$, $P=0.023)$, second surgery $(O R=7.021, P=0.004)$, bone window height $(\mathrm{OR}=6.543, P=0.041)$, and bone window area $(\mathrm{OR}=1.035, P=0.012)$ were independent risk factors for $\mathrm{CH}$ after $\mathrm{DC}$ surgery. It suggested that CT imaging technology could be utilized in the diagnosis of $\mathrm{CI}$. The risk factors of $\mathrm{CH}$ included coma time, SAH, GCS score no more than 8 , intraventricular hemorrhage, brain contusion, subdural hematoma, bilateral DC, bone window height, bone window area, and second surgery.
\end{abstract}

\section{Introduction}

Craniocerebral injury is a relatively common disease in neurosurgery [1]. Patients with CI are usually prone to hydrocephalus, and hydrocephalus will eventually cause disability or death in patients, thereby increasing mortality. Hydrocephalus has become an important cause of death in patients with CI [2]. Hydrocephalus refers to the breakdown of the balance between the formation and absorption of cerebrospinal fluid, which leads to the expansion of the ventricular system $[3,4]$. Clinically, it is usually divided into two types, communicative and non- $\mathrm{CH}$ [5]. The division of the two is mainly based on the difference in the blockage of the cerebrospinal fluid circulation pathway. If the horizontal position of the arachnoid particles is blocked, it is a $\mathrm{CH}$; but if the position above the arachnoid particles is blocked, it is a
non-CH. Such classification method is helpful for physicians to formulate appropriate clinical treatment plans for patients. DC is the first choice for the treatment of heavy CI [6]. DC has been widely adopted clinically, and the occurrence of hydrocephalus after DC surgery in the current application process has become a difficult problem in the treatment of CI. Therefore, the research on the changes of cerebrospinal fluid and the occurrence of hydrocephalus after DC surgery is very important for the clinical treatment of $\mathrm{CI}$ and the prognosis of patients.

With the rapid development of medical imaging technology, CT [7], MRI [8], and other imaging equipment can be utilized in the detection of patients with CI. Among them, a CT scan is currently the first choice for clinical diagnosis of $\mathrm{CI}$, which can quickly collect the information of the lesion area and clearly show the details of the intracranial injury. It 
can also reflect the relationship between the compensable spatial changes of the patient's CI and the development of the entire disease through a variety of changes in CT signs. It is a safe, noninvasive, and easy-to-operate inspection method, which is widely adopted clinically [9]. Lindberg et al. adopted CT to diagnose patients with traumatic brain injury. It was found that there were 111 patients including 81 cases of skull fractures, 27 cases of subdural hematoma, 24 cases of SAH, and 35 cases of other injuries [10]. Babl et al. suggested that the sensitivity of CT to identify clinical CI was 98.8\% (95\% CI: 95.6\%-99.8\%), and the specificity was $92.4 \%$ (95\% CI: 92.0\%-92.8\%) [11]. CT imaging technology can provide important guidance for the estimation of the disease and the selection of subsequent treatment measures and can provide an important diagnostic basis for neurosurgeons to evaluate the disease.

Based on the advantages of CT imaging technology, CT imaging technology was adopted to diagnose patients with $\mathrm{CI}$, to explore the relationship between $\mathrm{CI}$ and $\mathrm{CH}$, and the correlation between the CI DC surgery-related factors and the occurrence of $\mathrm{CH}$. It provided a theoretical basis for preventing and reducing the occurrence of $\mathrm{CH}$ after $\mathrm{CI}$ and the occurrence of $\mathrm{CH}$ after DC surgery.

\section{Materials and Methods}

2.1. Research Subjects and Grouping. 410 patients with traumatic brain injury who were admitted to the hospital from October 2015 to October 2019 were selected. Among them, 130 patients suffered from $\mathrm{CH}$, with 82 males and 48 females. Among the 410 patients, 116 were treated with DC, 28 of 116 patients had $\mathrm{CH}$, and the remaining 88 patients did not have $\mathrm{CH}$.

Inclusion criteria: (I) patients with a clear history of trauma; (II) patients who received craniocerebral CT examination; (III) patients with good image quality; and (IV) patients who received craniotomy in accordance with "Chinese Craniocerebral Injury Treatment Guidelines." Exclusion criteria: (I) patients who died 3 days after admission; (II) patients with hydrocephalus before injury; (III) patients with abandoned treatment in the middle of the process; (IV) patients with poor image quality; (V) patients with severe trauma; and (VI) patients with traumatic shock. This experiment had been approved by the ethics committee of the hospital, and the patients involved in the study had known and agreed with it.

2.2. CT Imaging Examination Method. GE 16-slice spiral CT instrument was adopted to perform head CT examination on all patients, the patients took the supine position, and CT plain scan was performed. The canthal ear line was taken as the scan line for CT examination, and the main parameters were set as follows. The voltage was $120 \mathrm{kV}$, the current was $150 \mathrm{mAs}$, the layer thickness was $0.8 \mathrm{~mm}$, the image reconstruction layer thickness was $4 \mathrm{~mm}$, the scanning pitch was $0.625 \mathrm{~mm}$, and the scanning matrix was $512 \times 512$. The patient's skull and soft tissue injuries were observed mainly through the bone window and soft tissue window. The image was then transferred to the postprocessing workstation, and the image was optimized via three-dimensional reconstruction and multiplane reconstruction technology.

2.3. Diagnostic Criteria for Hydrocephalus. CT imaging technology was applied to diagnose hydrocephalus. The main imaging feature was the enlarged frontal angle. There was a cap phenomenon near the lateral ventricle, the third and fourth ventricles, and the base pool could also be seen to expand. Hensson ventricular index was an important basis of the diagnostic criterion. The ratio of the distance between the frontal angle of the lateral ventricle on both sides of the caudate nucleus level and the distance between the inner plates of the skull at the same level was greater than $95 \%$ of the ratio for patients of the same age.

2.4. Observation Indexes. Patients were grouped and studied according to the diagnostic criteria of hydrocephalus, which were mainly divided into non- $\mathrm{CH}$ and $\mathrm{CH}$.

1) All patients were divided into 130 cases of $\mathrm{CH}$ (group A) and 280 cases of non-CH (group B). They were compared in terms of gender ratio, GCS score [12], and whether there was cerebral contusion and laceration, $\mathrm{SAH}$, diffuse axonal injury (DAI), intraventricular hemorrhage, subdural hemorrhage, skull fracture, and open CI.

2) 116 patients underwent decompression DC. According to whether $\mathrm{CH}$ occurred, 28 patients were $\mathrm{CH}$ (group $\mathrm{C}$ ) and 88 non- $\mathrm{CH}$ (group D). The relationship between postoperative hydrocephalus and surgical factors was analyzed and compared, which included bone window area, bone window height, secondary surgery ratio, early surgery ratio, unilateral/ bilateral DC surgery ratio, and early cranioplasty ratio.

Intraventricular hemorrhage was graded according to the Graeb scoring standards. According to the score results, it could be divided into mild, moderate, and severe. The score between 1 and 4 was mild, the score between 5 and 8 points was moderate, and the score between 9 and 12 points was severe. Minor hemorrhage in the lateral ventricle, bleeding volume no more than $1 / 2$ ventricle volume, bleeding volume more than $1 / 2$ ventricle volume, full of blood and enlarged were counted as 1, 2, 3, and 4 points, respectively. The intraventricular hemorrhage was normal in size, and the ventricles filled with hemorrhage and enlarged were counted as 1 and 2 points, respectively. The intraventricular hemorrhage was normal in size, and the ventricles filled with blood and enlarged were counted as 1 and 2 points, respectively.

The calculation method of bone window height $H$ and bone window area $S$ were as follows: the maximum diameter of the bone window was set as $D$, then $H$ was referred to the maximum vertical distance from the scalp of the research subject to the diameter $D$ on the CT plane of the maximum diameter $D$, and the calculation equation for $S$ was $S=\pi \times[2 \times(D / 2)+2 H]$. 
2.5. Statistical Analysis. SPSS20.0 statistical software was adopted for analysis. The measurement data were expressed as mean plus or minus standard deviation $(\bar{x} \pm s)$, which were tested by single-factor analysis of variance. The multiple linear regression methods were applied to analyze related factors. $P<0.05$ indicated statistical significance.

\section{Results}

3.1. CT Images of the Skull of Some Patients. The CT images of patients with CI are shown in Figure 1. It showed left frontal epidural hematoma; the main feature was a biconvex lensshaped high-density shadow on the inside of the skull, which was assessed as mild by GCS (Figure 1(a)). The subdural hematoma at the top of the left temporalis was mainly characterized by a certain degree of squeezing of the adjacent brain tissue, with split-brain and sulcus gradually narrowing and disappearing. Crescent-shaped high-density shadows could be seen on the inner part of the patient's skull, assessed by the GCS as moderate (Figure 1(b)). Figure 1(c) shows the left temporal lobe, frontal lobe subdural hematoma, cerebral contusion, and laceration, and the internal density of the brain contusion was uneven. The occurrence of low-density edema and high-density hemorrhage could be seen, assessed by the GCS as severe.

3.2. Analysis of Risk Factors for Hydrocephalus after CI. 410 patients with $\mathrm{CI}$ were divided into 130 cases of $\mathrm{CH}$ (group A) and 280 cases of non-CH (group B). The comparison of gender and age between group $A$ and group $B$ is shown in Figure 2. In group A, there were 86 patients aged 21-50 years, and 44 patients aged 51-70 years, including 82 males and 48 females. In group B, there were a total of 180 patients aged $21-50$, and a total of 100 patients aged 51-70, including 184 males and 96 females. The difference in gender and age of group A and group B patients was not greatly remarkable $(P>0.05)$.

The comparison results of coma time, GCS score, SAH, and DAI of group A and group B are shown in Figures 3 and 4. In Figure 3, the number of patients in group A with coma time less than $1 \mathrm{~W}$ and no less than $1 \mathrm{~W}$ was evidently more in contrast to that of group B $(P<0.01)$. In Figure 4, the difference in GCS scores between the two groups was greatly remarkable $(P<0.01)$. The number of SAH patients in group A was notably less in contrast to that of group B $(P<0.01)$. There was no observable difference in DAI between group A and group $\mathrm{B}(P>0.05)$.

The comparison of the clinical characteristics of the two groups of patients is shown in Figure 5. There were 74 and 45 patients with subdural hematoma in groups A and B, respectively. There were evidently more patients with subdural hematoma in group A than in group B $(P<0.01)$. Group A was with 76 cases of cerebral contusion and laceration and 19 cases of open CI. In group B, there were 234 cases of cerebral contusion and laceration and 52 cases of open CI. The number of patients with cerebral contusion and laceration and open $\mathrm{CI}$ in group $\mathrm{A}$ was notably less in contrast to that of group B $(P<0.01)$. In group A, there were 14 cases of mild,
48 cases of moderate, and 68 cases of severe intraventricular hemorrhage. In group B, there were 126 patients with mild intraventricular hemorrhage, 15 patients with moderate severity, and 62 patients with severe intraventricular hemorrhage. There was a greatly remarkable difference in the degree of intraventricular hemorrhage between the two groups $(P<0.01)$. However, there was no observable difference between group A and group B in terms of epidural hematoma, cerebrospinal fluid leakage, and linear skull fracture $(P>0.05)$.

Further logistic multiple regression analysis was performed on the factors with observable differences in the above results, as shown in Table 1. Coma time $(\mathrm{OR}=5.1283$, $P=0.001)$, SAH degree $(\mathrm{OR}=7.6543, P=0.020)$, GCS score no more than $8(\mathrm{OR}=3.5480, P=0.001)$, intraventricular hemorrhage ( $\mathrm{OR}=2.2653, P=0.003)$, scope of brain contusion $(\mathrm{OR}=1.036, P=0.002)$, and subdural hematoma $(\mathrm{OR}=2.4376, P=0.001)$ were independent risk factors for $\mathrm{CH}$.

3.3. Analysis of Risk Factors for Hydrocephalus after DC Surgery. Figure 6 shows the comparison results of early surgery, second surgery, and early cranioplasty between the two groups. In group C, there were 10, 8, and 4 patients with early surgery, second surgery, and early cranioplasty, accounting for $35.7 \%, 28.6 \%$, and $14.3 \%$, respectively. In group $\mathrm{D}$, there were 34,5 , and 29 patients with early surgery, second surgery, and early cranioplasty, accounting for $38.6 \%, 5.7 \%$, and $33.0 \%$, respectively. There was no observable difference in the proportion of early surgery between the two groups $(P>0.05)$. The proportion of secondary surgery in group $\mathrm{C}$ was evidently higher in contrast to that of group D $(P<0.01)$. The proportion of early cranioplasty in group $C$ was evidently lower in contrast to that of group $\mathrm{D}(P<0.05)$.

Figure 7 shows the comparison of the proportions of bilateral and unilateral DC between the two groups. There were 11 and 17 patients with bilateral DC and unilateral DC in group C, accounting for $39.3 \%$ and $60.7 \%$. There were 3 and 85 patients with bilateral DC and unilateral DC in group $\mathrm{D}$, accounting for $3.4 \%$ and $96.6 \%$. The proportion of bilateral DC in group $C$ was evidently higher in contrast to that of group $\mathrm{D}(P<0.05)$. The proportion of unilateral DC in group $\mathrm{C}$ was evidently lower in contrast to that of group $\mathrm{D}$, but it was not greatly remarkable $(P>0.05)$.

Figure 8 shows the comparison results of the two sets of bone window height and bone window area. Patients in group $\mathrm{C}$ had bone window height and bone window area of $3.2 \pm 0.7(\mathrm{~cm})$ and $138.2 \pm 15.2\left(\mathrm{~cm}^{2}\right)$, respectively. The bone window height and bone window area of patients in group D were $2.7 \pm 0.2(\mathrm{~cm})$ and $112.4 \pm 11.8\left(\mathrm{~cm}^{2}\right)$, respectively. The bone window height and bone window area of group $\mathrm{C}$ were evidently higher in contrast with those of group $\mathrm{D}(P<0.01)$.

Further logistic multiple regression analysis was performed on the factors with observable differences in the above results, as shown in Table 2. It was concluded that that bilateral $\mathrm{DC}(\mathrm{OR}=15.342, P=0.023)$, secondary surgery $(\mathrm{OR}=7.021, P=0.004)$, bone window height $(\mathrm{OR}=6.543$, 


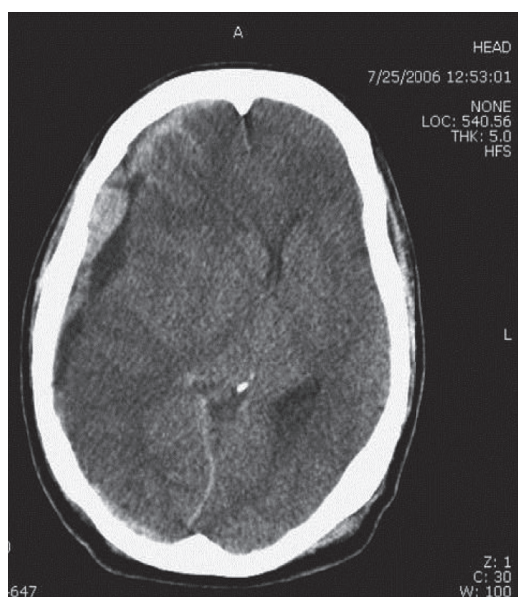

(a)

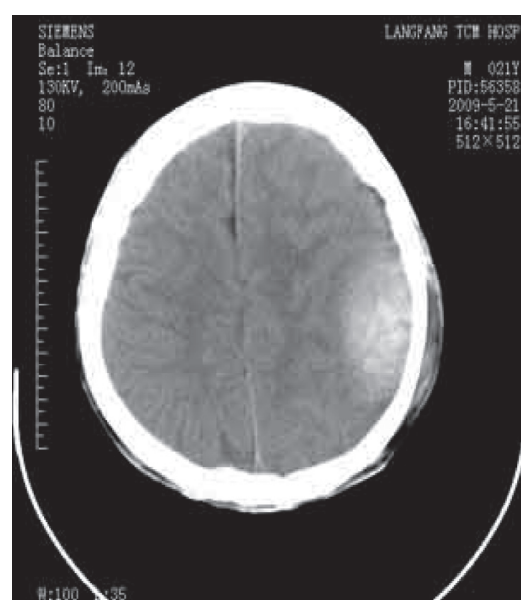

(b)

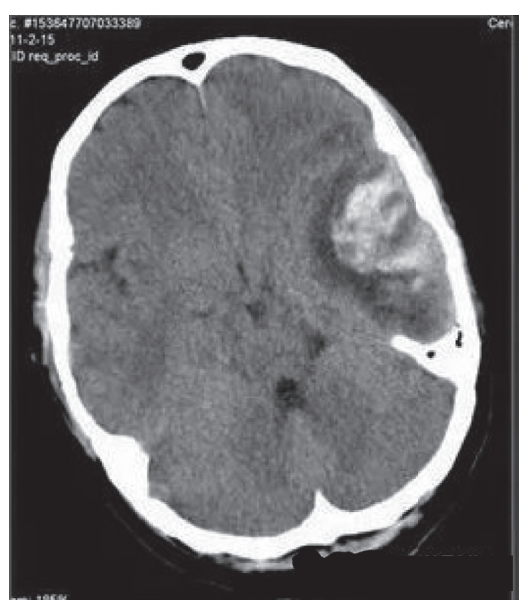

(c)

FIgURE 1: CT images of patients with CI. (a) Epidural hematoma; (b) subdural hematoma; (c) subdural hematoma with cerebral contusion and laceration.

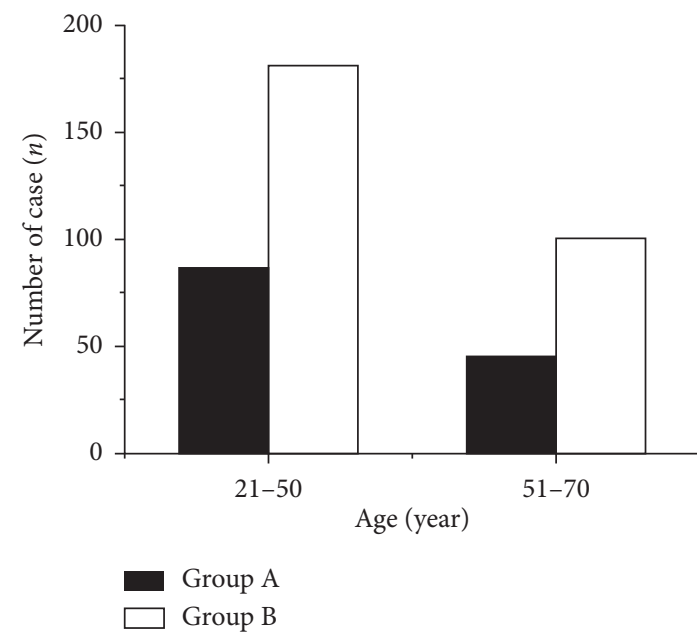

(a)

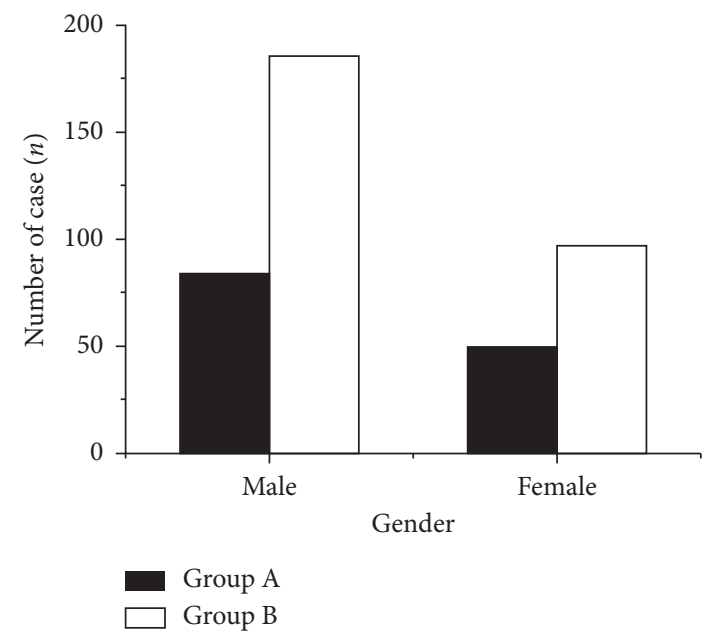

(b)

Figure 2: Comparison of gender and age between the two groups. (a) Age and (b) gender.

$P=0.041)$, and bone window area $(\mathrm{OR}=1.035, P=0.012)$ were independent risk factors for the occurrence of $\mathrm{CH}$ after DC surgery.

\section{Discussion}

Hydrocephalus is a common clinical symptom after CI, which usually refers to the accumulation of large amounts of cerebrospinal fluid caused by abnormal brain effusion production or absorption in the subarachnoid space and around the ventricular system, leading to a variety of complex clinical syndromes [13]. Studies revealed that the lower the GCS score, the longer the coma time, the more likely to develop hydrocephalus [7]. This may be because patients with a low GCS score and a long coma time usually have more severe cerebral contusion and laceration, SAH, and intracranial hematoma. The cerebral venous circulation is blocked, and the brain tissue may shrink due to damage, which leads to the appearance of the expansion of the ventricular system. As a result, the incidence of hydrocephalus increased. Moreover, intraventricular hemorrhage and subdural hematoma are also high-risk factors for hydrocephalus [14]. Chen et al. suggested that, compared with brain injury patients without traumatic SAH, the risk of hydrocephalus in patients with traumatic SAH was about three times [15]. This study showed the coma time, the degree of SAH, the GCS score no more than 8, intraventricular hemorrhage, brain contusion, and subdural hematoma were independent risk factors for $\mathrm{CH}$, which was consistent with the results of Hutchinson et al. [16].

DC surgery is an effective clinical treatment method for CI [17]. In patients with CI DC, posttraumatic hydrocephalus is very common. However, its incidence, mechanism, and risk factors have not yet been fully elucidated. This 


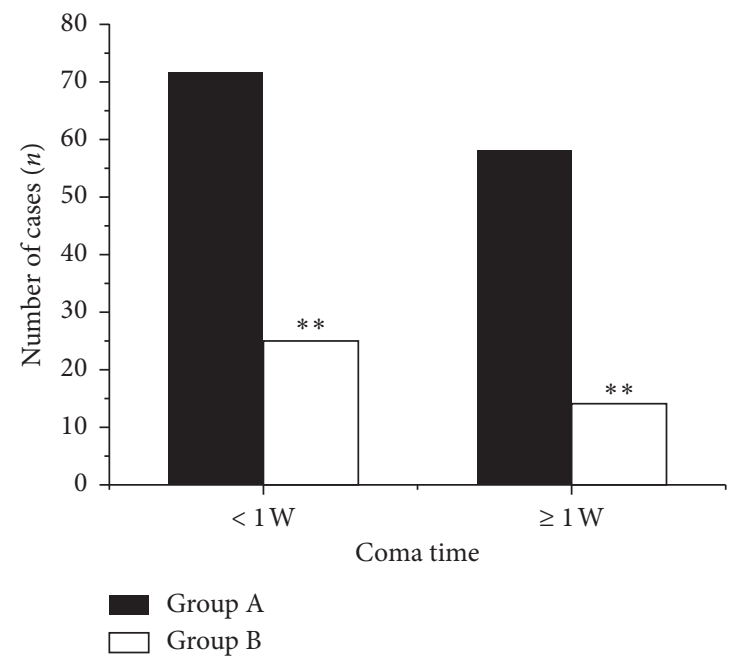

Figure 3: Comparison of coma time between the two groups. ${ }^{* *}$ compared to group A, $P<0.01$.

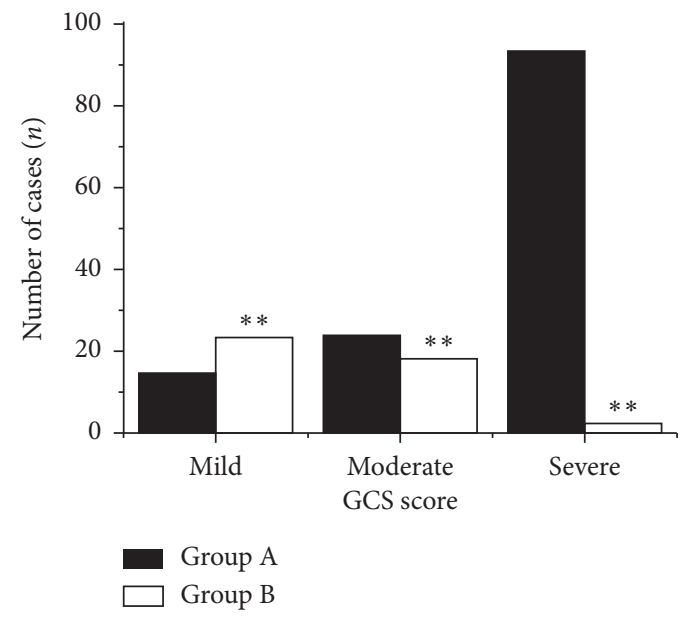

(a)

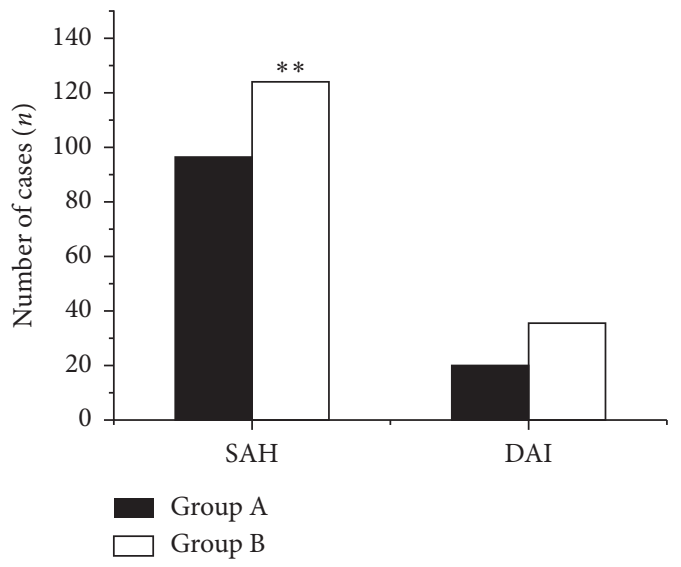

(b)

Figure 4: Comparison of GCS score, SAH, and DAI between the two groups. (a) GCS score; (b) SAH and DAI; ** compared with group, A $P<0.01$.

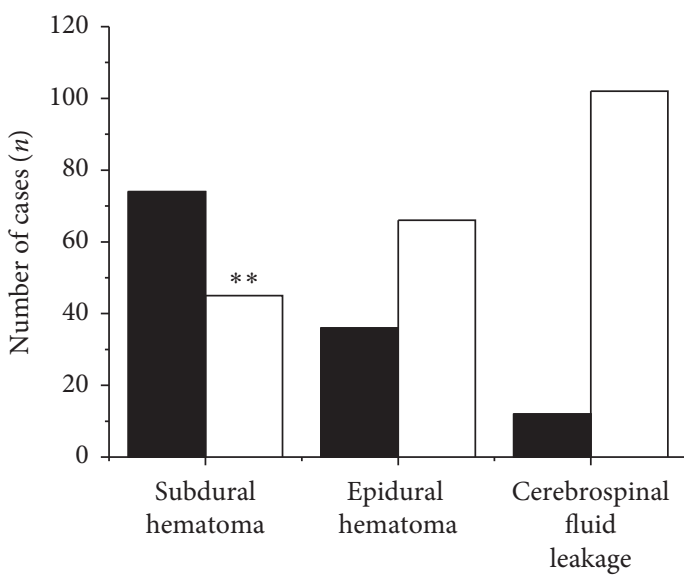

Group A Group B

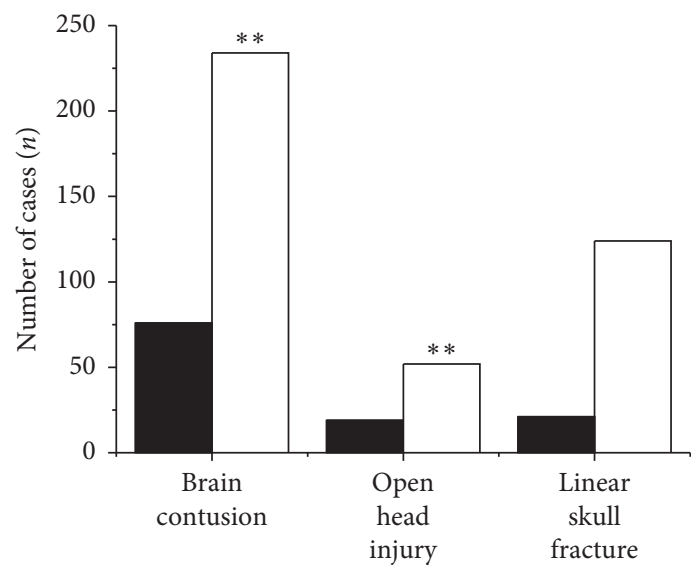

Group A Group B

(a)

Figure 5: Continued. 


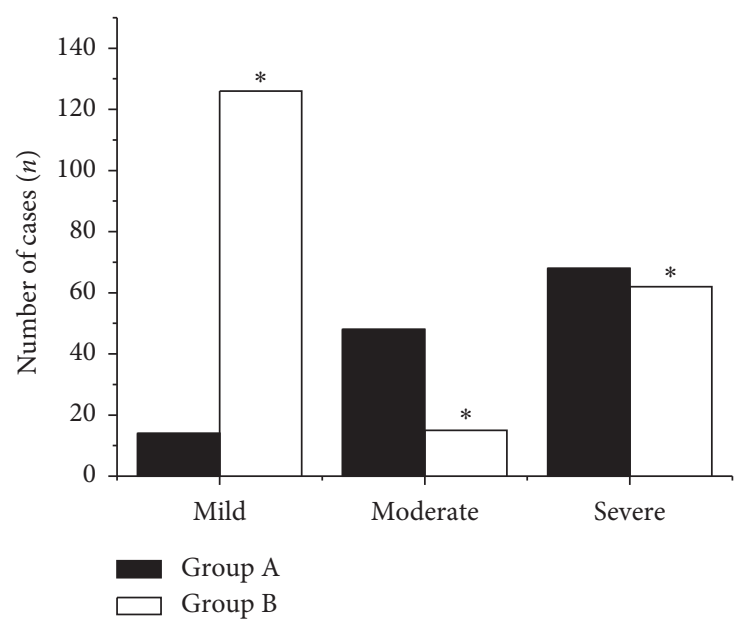

(c)

FiguRE 5: Comparison of clinical characteristics of patients with CI. (a) Subdural hematoma, epidural hematoma, and cerebrospinal fluid leakage; (b) cerebral contusion and laceration, open CI, and linear skull fracture; and (c) degree of intraventricular hemorrhage. *and ** compared to group A, $P<0.05$ and $P<0.01$, respectively.

TABle 1: Analysis of risk factors of $\mathrm{CH}$ after CI.

\begin{tabular}{|c|c|c|c|c|c|c|}
\hline \multirow{2}{*}{ Factors } & \multirow{2}{*}{$\mathrm{B}$} & \multirow{2}{*}{$\mathrm{SE}$} & \multirow{2}{*}{ OR } & \multicolumn{2}{|c|}{$95 \% \mathrm{CI}$} & \multirow{2}{*}{$P$} \\
\hline & & & & Lower limit & Upper limit & \\
\hline Coma time & 1.5387 & 0.7754 & 5.1283 & 3.2543 & 14.2653 & $0.001^{*}$ \\
\hline SAH degree & 2.0765 & 0.8354 & 7.6543 & 4.1765 & 38.3652 & $0.020^{*}$ \\
\hline GCS score no more than 8 & 1.5628 & 0.6429 & 3.5480 & 3.8762 & 17.3652 & $0.001^{*}$ \\
\hline Intraventricular hemorrhage & 1.2543 & 0.4276 & 2.2653 & 2.1865 & 36.2653 & $0.003^{*}$ \\
\hline Brain contusion scope & 0.0486 & 0.0023 & 1.036 & 1.0275 & 6.2738 & $0.002^{*}$ \\
\hline Subdural hematoma & 0.0654 & 0.7276 & 2.4376 & 1.8652 & 18.3654 & $0.001^{*}$ \\
\hline
\end{tabular}

*denotes $P<0.05$.

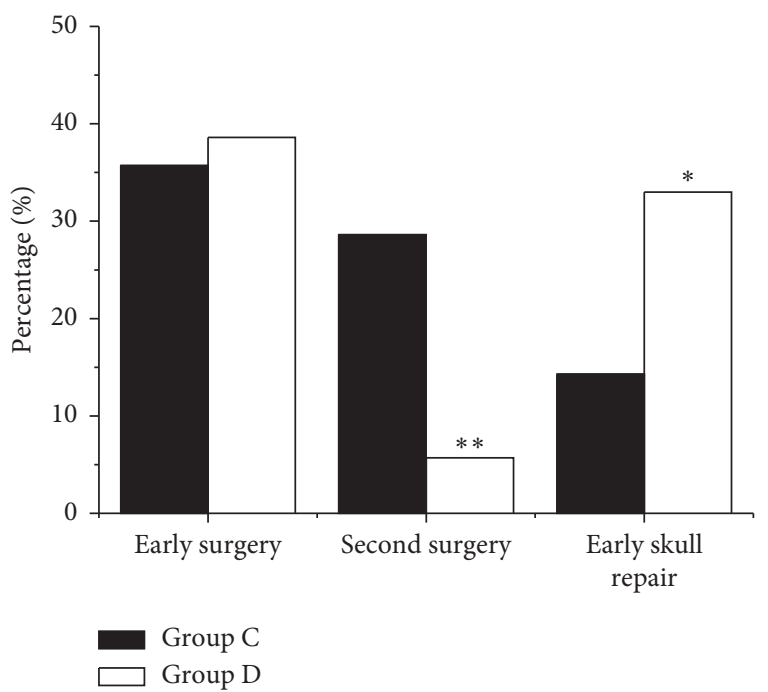

FIgURE 6: Comparison of early surgery, second surgery, and early skull repair. ${ }^{*}$ and ${ }^{* *}$ mean, compared to group $C, P<0.05$ and $P<0.01$, respectively.

study aimed to determine the incidence of PTH and related factors. The results showed bilateral DC, second surgery, bone window height, and bone window area were independent risk factors for $\mathrm{CH}$ after DC surgery. The reasons

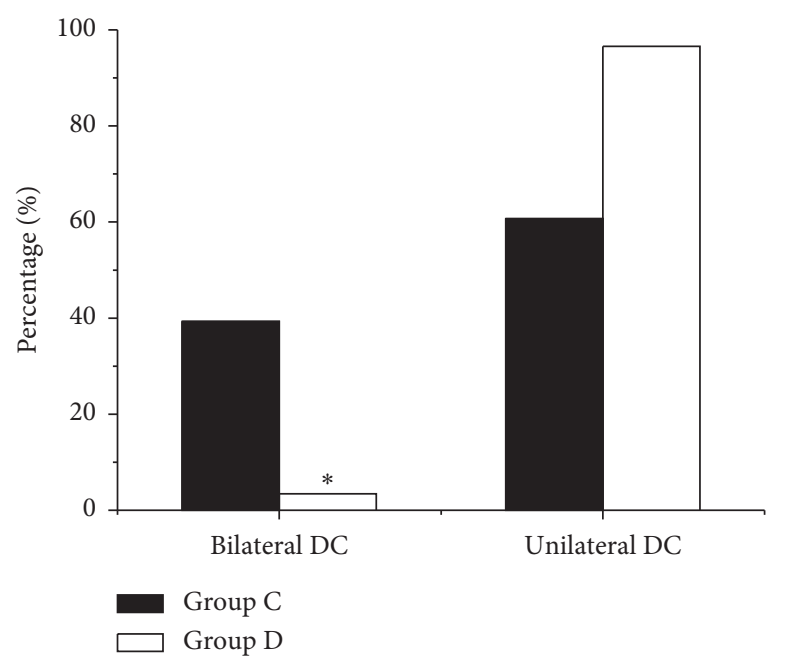

FIGURE 7: Comparison of the proportions of unilateral DC and bilateral DC in the two groups. *means, compared to group C, $P<0.05$.

may be that the loss of skull protection after DC surgery caused the imbalance of intracranial pressure, and the cerebrospinal fluid pressure was affected, which had a greater impact on the generation and absorption of cerebrospinal 


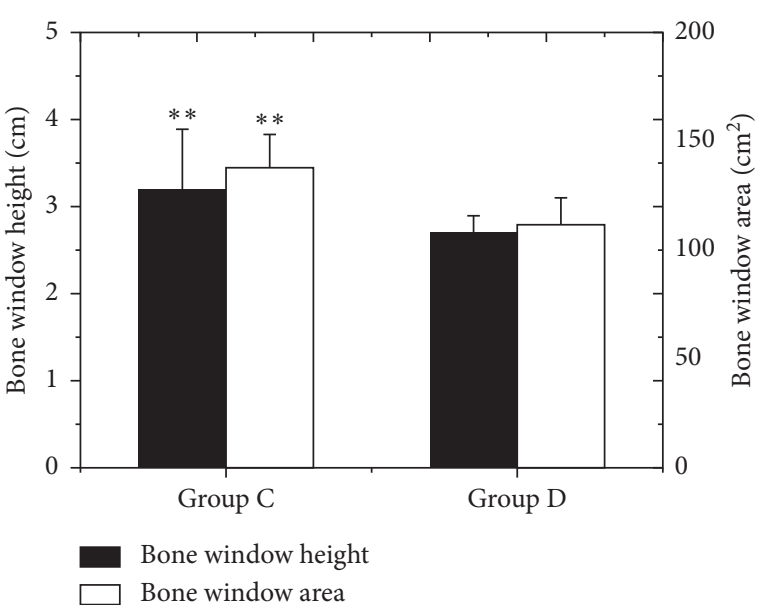

Figure 8: Comparison of bone window height and bone window area of the two groups. * means, Compared to group C, $P<0.05$.

TABLE 2: Analysis of risk factors of $\mathrm{CH}$ after CI DC.

\begin{tabular}{lcccc}
\hline \multirow{2}{*}{ Factors } & \multirow{4}{c}{$95 \%$ CI } & \multirow{2}{*}{$P$} \\
& & Lower limit & Upper limit & \\
\hline Bilateral DC & 15.342 & 1.563 & 123.38 & $0.023^{*}$ \\
Second surgery & 7.021 & 1.652 & 30.762 & $0.004^{*}$ \\
Bone window height & 6.543 & 1.872 & 23.653 & $0.041^{*}$ \\
Bone window area & 1.035 & 1.021 & 1.121 & $0.012^{*}$ \\
\hline
\end{tabular}

*denotes $P<0.05$.

fluid. Or, it was because that the bone window height and bone window area of different sizes had different effects on venous return. Patients undergoing secondary surgery generally had rebleeding, severe brain tissue damage, large and numerous bone windows, and a notable increase in hydrocephalus. Di et al. analyzed the risk factors of hydrocephalus after CI DC, and it was found that bilateral DC, intraventricular hemorrhage, and GCS score less than 6 were important predictors of hydrocephalus after DC surgery [18], which was also consistent with the results of this article.

\section{Conclusion}

CT imaging technology was applied to the diagnosis of patients with CI. By analyzing the influencing factors of $\mathrm{CH}$ after CI DC, it was found that coma time, SAH degree, GCS score no more than 8 , intraventricular hemorrhage, brain contusion, and subdural hematoma were closely related to the occurrence of $\mathrm{CH}$. Moreover, bilateral DC, secondary surgery, bone window height, and bone window area were independent risk factors for $\mathrm{CH}$ after DC surgery. However, there are still some shortcomings in this study. For example, the sample size is small and the number of influencing factors of $\mathrm{CH}$ included is limited. It is necessary to increase the number of samples and further analyze the risk factors of $\mathrm{CH}$ after DC surgery. In short, the results can provide a theoretical basis for the analysis of the causes of hydrocephalus after DC surgery, the prevention of hydrocephalus after $\mathrm{CI}$, and subsequent intervention treatment.

\section{Data Availability}

No data were used to support this study.

\section{Conflicts of Interest}

The authors declare that they have no conflicts of interest.

\section{References}

[1] K. K. Wang, Z. Yang, and T. Zhu, "An update on diagnostic and prognostic biomarkers for traumatic brain injury," Expert Review of Molecular Diagnostics, vol. 18, no. 2, pp. 165-180, 2018.

[2] R. Fattahian, S. R. Bagheri, and M. Sadeghi, "Development of posttraumatic hydrocephalus requiring ventriculoperitoneal shunt after decompressive craniectomy for traumatic brain injury: a systematic review and meta-analysis of retrospective studies," Medical Archives, vol. 72, no. 3, pp. 214-219, 2018.

[3] R. A. Dorner, V. J. Burton, M. C. Allen, S. Robinson, and B. P. Soares, "Preterm neuroimaging and neurodevelopmental outcome: a focus on intraventricular hemorrhage, posthemorrhagic hydrocephalus, and associated brain injury," Journal of Perinatology, vol. 38, no. 11, pp. 1431-1443, 2018.

[4] H. M. Makinde, T. B. Just, C. M. Cuda, N. Bertolino, D. Procissi, and S. J. Schwulst, "Monocyte depletion attenuates the development of posttraumatic hydrocephalus and preserves white matter integrity after traumatic brain injury," PLoS One, vol. 13, no. 11, Article ID e0202722, 2018.

[5] A. Agarwal, G. Bathla, and S. Kanekar, "Imaging of communicating hydrocephalus," Seminars in Ultrasound CT and $M R$, vol. 37, no. 2, pp. 100-108, 2016.

[6] D. A. Brown and E. F. Wijdicks, "Decompressive craniectomy in acute brain injury," Handbook of Clinical Neurology, vol. 140, pp. 299-318, 2017.

[7] D. Pavlovic, S. Pekic, M. Stojanovic, and V. Popovic, "Traumatic brain injury: neuropathological, neurocognitive and neurobehavioral sequelae," Pituitary, vol. 22, no. 3, pp. 270-282, 2019.

[8] J. K. Yue, E. L. Yuh, and F. K. Korley, “Association between plasma GFAP concentrations and MRI abnormalities in patients with CT-negative traumatic brain injury in the TRACKTBI cohort: a prospective multicentre study," The Lancet Neurology, vol. 18, no. 10, pp. 953-961, 2019.

[9] A. T. Hale, D. P. Stonko, and A. Brown, "Machine-learning analysis outperforms conventional statistical models and CT classification systems in predicting 6-month outcomes in pediatric patients sustaining traumatic brain injury," $\mathrm{Neu}$ rosurgical Focus, vol. 45, no. 5, Article ID E2, 2018.

[10] D. M. Lindberg, N. V. Stence, and J. A. Grubenhoff, "Feasibility and accuracy of fast MRI versus CT for traumatic brain injury in young children," Pediatrics, vol. 144, no. 4, Article ID e20190419, 2019.

[11] F. E. Babl, E. Oakley, and S. R. Dalziel, "Accuracy of clinician practice compared with three head injury decision rules in children: a prospective cohort study," Annals of Emergency Medicine, vol. 71, no. 6, pp. 703-710, 2018.

[12] P. M. Brennan, G. D. Murray, and G. M. Teasdale, "Simplifying the use of prognostic information in traumatic brain injury. part 1: the GCS-pupils score: an extended index of clinical severity," Journal of Neurosurgery, vol. 128, no. 6, pp. 1612-1620, 2018.

[13] D. Nasi, M. Dobran, and A. Di Rienzo, "Decompressive craniectomy for traumatic brain injury: the role of 
cranioplasty and hydrocephalus on outcome," World Neurosurgery, vol. 116, pp. e543-e549, 2018.

[14] T. Zhao, W. Ouyang, S. Wang, and P. Wu, "Overdrainage secondary to ventriculosinus shunt," World Neurosurgery, vol. 102, pp. 696.e17-696.e20, 2017.

[15] K.-H. Chen, C.-P. Lee, and Y.-H. Yang, "Incidence of hydrocephalus in traumatic brain injury," Medicine, vol. 98, no. 42, Article ID e17568, 2019.

[16] P. J. Hutchinson, A. G. Kolias, and I. S. Timofeev, "Trial of decompressive craniectomy for traumatic intracranial hypertension," New England Journal of Medicine, vol. 375, no. 12, pp. 1119-1130, 2016.

[17] G. Di, Y. Zhang, and H. Liu, "Postoperative complications influencing the long-term outcome of head-injured patients after decompressive craniectomy," Brain and Behavior, vol. 9, no. 1, Article ID e01179, 2019.

[18] G. Di, Q. Hu, D. Liu, X. Jiang, J. Chen, and H. Liu, "Risk factors predicting posttraumatic hydrocephalus after decompressive craniectomy in traumatic brain injury," World Neurosurgery, vol. 116, pp. e406-e413, 2018. 